\title{
New Efficient Method for Coding Color Images
}

\author{
Walaa M.Abd-Elhafiez, Wajeb Gharibi
}

\begin{abstract}
In this paper a novel color image compression technique for efficient storage and delivery of data is proposed. The proposed compression technique started by $\mathrm{RGB}$ to $\mathrm{YCbCr}$ color transformation process. Secondly, the canny edge detection method is used to classify the blocks into the edge and non-edge blocks. Each color component $\mathrm{Y}, \mathrm{Cb}$, and $\mathrm{Cr}$ compressed by discrete cosine transform (DCT) process, quantizing and coding step by step using adaptive arithmetic coding. Our technique is concerned with the compression ratio, bits per pixel and peak signal to noise ratio, and produce better results than JPEG and more recent published schemes (like CBDCT-CABS and MHC). The provided experimental results illustrate the proposed technique that is efficient and feasible in terms of compression ratio, bits per pixel and peak signal to noise ratio.
\end{abstract}

Keywords - Image compression, color image, Q-coder, quantization, edge-detection.

\section{INTRODUCTION}

$\mathrm{I}_{\mathrm{i}}^{\mathrm{N}}$ the recent years there has been an astronomical increase in the usage of computers for a variety of tasks. One of the most common usages has been the storage, manipulation, and transfer of digital images. The files that comprise these images, however, can be quite large and can quickly take up precious memory space on the computer's hard drive. In multimedia application, most of the images are in color. Color images contain a lot of data redundancy and require a large amount of storage space.

Image compression refers to the reduction of the size of the data that images contain. Image compression schemes in [1], [2] exploit certain data redundancies to convert the image to a smaller form. A typical image compression system is shown in Fig. 1. The data reduction, or compression, is performed by a device known as the encoder. The encoder reduces the data size of the original image A. The compressed image B is the output that passes through a channel (usually an actual transmission channel or a storage system) to the decoder. The decoder reconstructs, or decompresses, the image $\mathrm{C}$ from the compressed data. The ratio of the size (amount of data or bandwidth) of the original image to the size of the compressed image is known as the compression ratio or compression rate. The compression ratio can also be expressed in bpp (bits per pixel). The term bit rate is a general term for bpp. The higher the compression rate, the greater is the reduction of data [3], [4]. Depending on the application, the channel may be affected by the noise that results in distortion of the compressed image during transmission. If so, the channel is known as an errorprone channel; otherwise, it is errorless. In Fig. 1, the channel

Walaa M. Abd-Elhafiez and Wajeb Gharibi are with College of Computer Science \& Information Systems, Jazan University, Jazan, Kingdom of Saudi Arabia. (e-mail: walaa.hussien@science.sohag. edu. eg; gharibiw@hotmail .com). is assumed to be error-free. Hence, $\mathrm{B}$ is the input to the decoder. Data compression schemes can be divided into two broad classes:

1. Lossless compression schemes [5], [6], in which $\mathrm{C}$ is identical to A.

2. Lossy compression schemes [7], [8], which provide much higher compression than lossless compression but allow $\mathrm{C}$ to be different from $\mathrm{A}$.

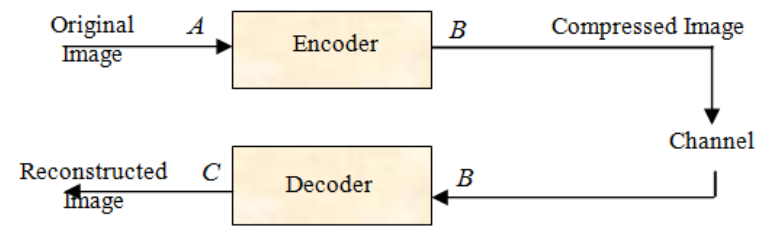

Fig. 1 Block diagram of image compression system

This paper is structured as follows: Section II reviews some related work. Our technique is described in Section 3. Experimental results are shown in Section IV. Conclusions are drawn in Section V.

\section{RELATED WORK}

Wang et.al [9] presented a cost effective block truncation coding (CE-BTC) using low-cost approach for color image compression. The usage of line buffer memory in low-cost approach CE-BTC is only a half of that in the optimal approach in CE-BTC. Therefore, the low-cost approach CEBTC can be suited to apply to some restrained resource applications such as frame memory reduction in LC Displays (LCD) overdrive. Simulation results show that the proposed CE-BTC outperforms the VQ-BTC in PSNR up to $3 \mathrm{~dB}$ and much better subject visual quality. Sowmyan et.al [10] have been proposed several methods for color image compression but the reconstructed image have very low signal to noise ratio that made it inefficient. Their technique worked on the spatial domain where the pixel values of RGB planes of the input color image is mapped onto two-dimensional planes. Satish and Shishir [11] used various contemporary standards by Joint Picture Expert Group for compression. They exploited the correlation among the color components using a component color space transform before the subband transform stage. The transforms used to decorrelate the colors are primarily the fixed kernel transforms, which are not suitable for the large class of images. In their paper an image dependent color space transform (ID-CCT), exploiting the inter-channel redundancy optimally and which is very much suitable for compression proposed. Also, the comparative performance has been evaluated, and a significant improvement has been observed, objectively as well as subjectively over other quantifiable methods. 


\section{The Proposed Color IMAge COMPRESSION Method}

Each color image consists of three components whether stored in $\mathrm{RGB}$ or $\mathrm{YCbCr}$ format. For this application, the input image is first converted into $\mathrm{YCbCr}$ format as in [12], because, in this way, additional decorrelation of the components (better compression) is done. Then classified the image into the background and foreground portions as describe in section 3.2. Then the image is subdivided into $8 \times 8$ blocks, and DCT coefficients are computed for each block. The quantization is performed conferring to predetermined quantization table. The quantized values are then rearranged according to zig-zag scan arrangement. The less important values are discarded (as describe in section 3.3) from the list in the zig-zag arrangement. After discarding insignificant coefficients, the remaining coefficients are compressed by the adaptive arithmetic coding (Q-coder).

\section{Algorithm}

1. Input the image to be compressed.

2. Classify the input image into background and foreground based on edges.

3. Subdivide the input image into $8 \times 8$ blocks

4. Find the DCT coefficients for each block.

5. Quantize the DCT coefficients based on predetermined quantization table.

6. Apply the modification of the quantized coefficient based on the classification in step 2.

7. Assemble the blocks into a continuous stream.

8. Compress the resulting values by apply Q-coder.

\section{A. Classification Step}

Edges often occur at points where there is a large variation in the luminance values in the image, and consequently they often indicate the edges, or occluding boundaries, of the object in the scene. There are several techniques have been used for edge detection [13]. In this paper, Canny Method is used. The canny edge detection algorithm is known to many as the optimal edge detector [14]. The image is divided into two classes: edge blocks and non-edge blocks.

\section{B. The Modification}

To increase compression ratio, in this part we suggested technique modification of JPEG compression by collection between JPEG compression technique and edge extraction. The modification will be done after the quantization step. The image is subdivided into a block of pixels, and then these blocks are classified into the edge (significant regions/foreground) and non-edge (insignificant regions/background) blocks. In the first method (A-1), the non-edge blocks are compressed using the DC coefficient only and all significant coefficients are used for the edge blocks. In the second method (A-2) on each component ( $\mathrm{Y}, \mathrm{Cb}$, and $\mathrm{Cr}$ ), the DC coefficient only is used for coding the non-edge blocks (insignificant regions). 70\% (choose by experimental) of the non-zero quantized $\mathrm{AC}$ coefficients have been used in the coding of edge blocks. In the third method (A-3) on each component $(\mathrm{Y}, \mathrm{Cb}$, and $\mathrm{Cr})$, the $\mathrm{DC}$ coefficient only is used for coding the non-edge blocks (insignificant regions). 50\% of the non-zero quantized AC coefficients have been used in the coding of edge blocks.

\section{Q-Coder (Adaptive Arithmetic Code)}

Adaptive arithmetic code [15] is a lossless compression technique that benefits from treating multiple symbols as a single data unit but at the same time retains the incremental symbol-by-symbol coding approach of Huffman coding. Arithmetic coding separates the coding from the modeling. This process allows for the dynamic adaptation of the probability model without affecting the design of the coder. Provisions for substituting Huffman coding for arithmetic coding are contained in many of the image compression standards.

\section{EXPERIMENTAL RESULTS}

For the implementation and evaluation of the algorithms, we developed a MATLAB code and performed the testing on a standard color test images Lena, Fruit, and Airplane of size $512 \times 512$ and another test image Zelda and House of size 256x256 (Fig. 2). We analyze the results obtained with the first, second, and finally, the proposed algorithm. All images and tables from the experiments are given. Standard measures for image compression [16], like compression ratio (CR) and peak signal to noise ratio (PSNR) were used, which are calculated for comparing the performance of the proposed method as per the following representations:

$$
\mathrm{CR}=\frac{\text { Orginal image size in bytes }}{\text { Compressed image size in bytes }}
$$

$$
\mathrm{PSNR}=10 \times \log _{10} \frac{255^{2} \times 3}{\operatorname{MSE}(\mathrm{Y})+\mathrm{MSE}(\mathrm{Cb})+\mathrm{MSE}(\mathrm{Cr})}
$$

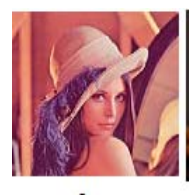

$\stackrel{a}{w}$

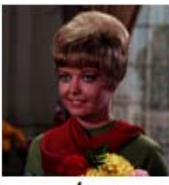

b

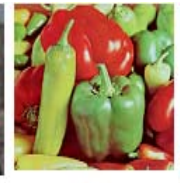

c

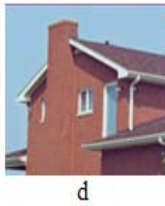

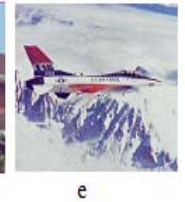

Fig. 2 Original test images: (a) Lena, (b) Zelda, (c) Fruit, (d) House and (e) Airplane

From the results listed in Table I, the proposed codec achieves a high performance, and we can conclude that about $0.1299-0.3404$-bit rate reduction on average is achievable by using the proposed method (A-3). The subjective visual quality is compared using a different color image, as shown in Figs. 3, 4, 5, respectively. Figs. 3 (c), 4 (c), and 5 (c) show less block artifact than resulted image by A-3 with block size $8 \times 8$ in Figs. 3 (a), 4 (a), and 5 (a).

Experiment results show that A-1 and A-2 perform better in PSNR compared with A-3. Although A-1 shows a litter better performance than A-2, the bit rate of A-1 is much higher than that of A-2. 

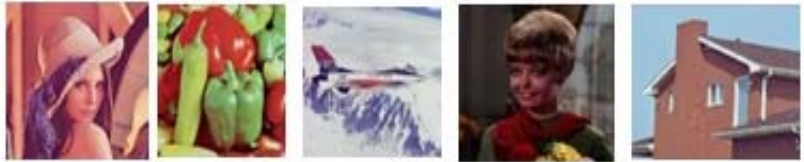

(a)
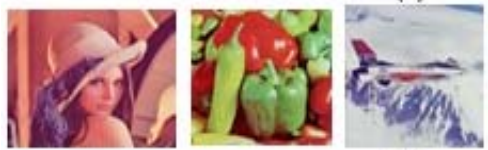

(b)
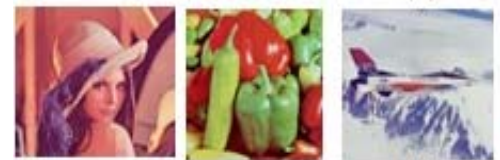

(c)
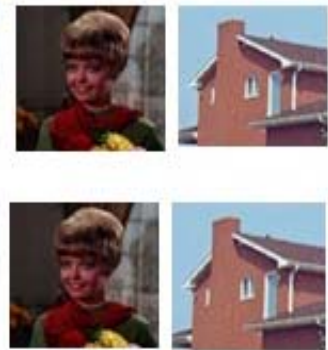

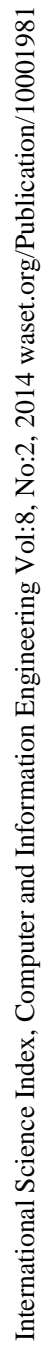

Fig. 3 Compressed images visual performance of the proposed method (A-1): a) DCT block size is $8 \times 8$, b) DCT block size is $16 \times 16$ and c) DCT block size is $32 \times 32$
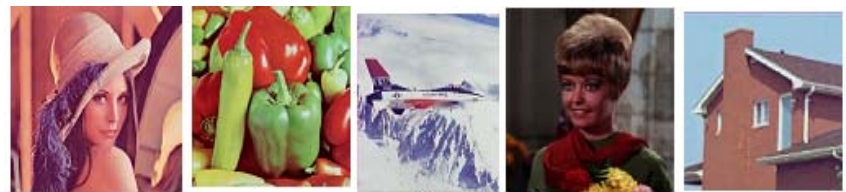

(a)
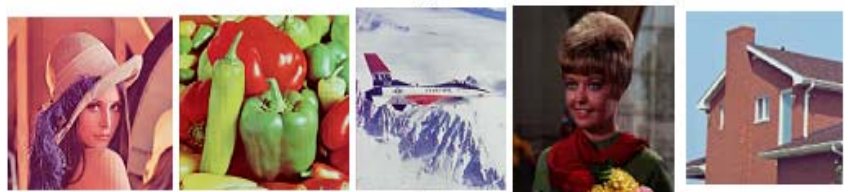

(b)
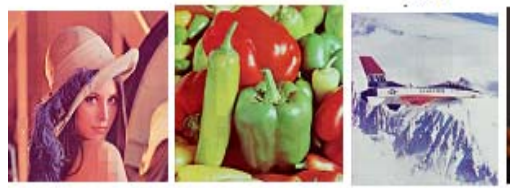

(c)
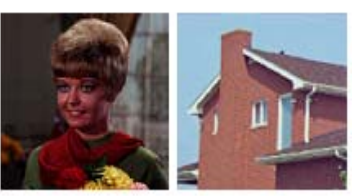

Fig. 4 Compressed images visual performance of the proposed method (A-2): a) DCT block size is $8 \times 8$, b) DCT block size is $16 \times 16$ and c) DCT block size is $32 \times 32$

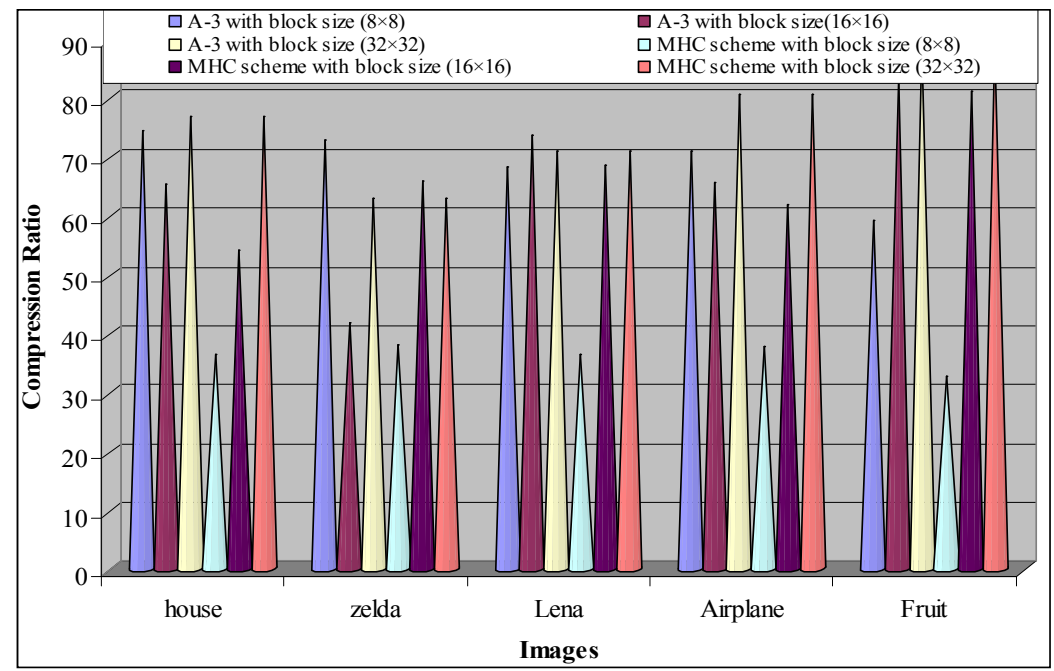

Fig. 6 Compression ratios attained for test images with different block size of the proposed method (A-3) and MHC scheme [17] 


\section{V.CONCLUSIONS}

This paper analyzes the color images and identifies the most important contexts between the luminance component and the chrominance components based on the analysis, a new color image coding scheme is proposed and evaluated. Significant bit rate reduction has been achieved by employing classification step.
Simulation results show that the proposed method outperforms the JPEG in PSNR up to $2.27 \mathrm{~dB}$ and much better subject visual quality. Comparing with CBDCT-CABS, the proposed method also performs a better in subjective visual quality and in PSNR by $2.83 \mathrm{~dB}$ on average. As demonstrated the best compromise results between bpp and PSNR are obtained for block size $8 \times 8$ for proposed techniques.

TABLE I

Performance Evaluation of the Proposed Methods

\begin{tabular}{|c|c|c|c|c|c|c|c|c|c|}
\hline \multicolumn{10}{|c|}{ BLOCK SIZE $(8 \times 8)$} \\
\hline \multirow{2}{*}{ IMAGE } & \multicolumn{3}{|c|}{ A-1 } & \multicolumn{3}{|c|}{ A-2 } & \multicolumn{3}{|c|}{ A-3 } \\
\hline & Bpp & PSNR & CR & Bpp & PSNR & CR & Bpp & PSNR & CR \\
\hline House & 0.4236 & 35.240 & 56.6634 & 0.356 & 34.362 & 67.3430 & 0.322 & 33.938 & 74.5292 \\
\hline Zelda & 0.4674 & 35.379 & 51.3504 & 0.374 & 34.580 & 64.0704 & 0.329 & 34.029 & 72.8886 \\
\hline Lena & 0.450 & 34.746 & 53.2367 & 0.385 & 33.998 & 62.1937 & 0.351 & 33.544 & 68.2333 \\
\hline Airplane & 0.5018 & 36.315 & 47.8289 & 0.392 & 35.198 & 61.2218 & 0.338 & 34.502 & 70.9736 \\
\hline Fruit & 0.552 & 34.183 & 43.4682 & 0.456 & 33.739 & 52.5980 & 0.405 & 33.405 & 59.1970 \\
\hline Average & 0.4789 & 35.1726 & 50.50952 & 0.3926 & 34.3754 & 61.48538 & 0.349 & 33.8836 & 69.16434 \\
\hline \multicolumn{10}{|c|}{ BLOCK SIZE $(16 \times 16)$} \\
\hline House & 0.6631 & 38.3959 & 36.1961 & 0.4685 & 36.5021 & 51.2233 & 0.3668 & 35.1075 & 65.4270 \\
\hline Zelda & 1.1599 & 37.2084 & 20.6910 & 0.7764 & 36.1707 & 30.9114 & 0.5732 & 34.9759 & 41.8738 \\
\hline Lena & 0.5290 & 36.5823 & 45.3716 & 0.4035 & 35.6310 & 59.4734 & 0.3263 & 34.5722 & 73.5439 \\
\hline Airplane & 0.7369 & 39.2232 & 32.5705 & 0.4938 & 37.1619 & 48.6026 & 0.3660 & 35.5585 & 65.5722 \\
\hline Fruit & 0.5340 & 34.7061 & 44.9428 & 0.3734 & 34.1404 & 64.2661 & 0.2882 & 33.5433 & 83.2765 \\
\hline Average & 0.7245 & 37.2231 & 35.9544 & 0.5031 & 35.9212 & 50.89536 & 0.3841 & 34.7514 & 65.93868 \\
\hline \multicolumn{10}{|c|}{ BLOCK SIZE $(32 \times 32)$} \\
\hline House & 0.6112 & 38.9130 & 39.2656 & 0.4145 & 36.6280 & 57.8983 & 0.3122 & 34.9308 & 76.8713 \\
\hline Zelda & 0.7188 & 37.1556 & 33.3913 & 0.5049 & 36.1446 & 47.5329 & 0.3822 & 35.0267 & 62.7915 \\
\hline Lena & 0.6688 & 36.5637 & 35.8841 & 0.4561 & 35.5973 & 52.6151 & 0.3379 & 34.4493 & 71.0289 \\
\hline Airplane & 0.5907 & 38.9460 & 40.6294 & 0.4023 & 36.9724 & 59.6556 & 0.2983 & 35.3787 & 80.4524 \\
\hline Fruit & 0.3443 & 34.1088 & 69.6991 & 0.3133 & 34.1664 & 76.5999 & 0.2697 & 33.9348 & 88.9843 \\
\hline Average & 0.5867 & 37.1374 & 43.7739 & 0.4182 & 35.9017 & 58.86036 & 0.3200 & 34.7440 & 76.02568 \\
\hline
\end{tabular}

\begin{tabular}{c|c|c|c|c|c|c|c|c}
\multirow{2}{*}{ IMAGE } & \multicolumn{3}{|c}{ JPEG } & \multicolumn{3}{c}{ Proposed method (A-3) } & \multicolumn{3}{c}{ CBDCT-CABS [18] } \\
\cline { 2 - 8 } & bpp & PSNR & bpp & PSNR & CR & bpp & PSNR & CR \\
\hline
\end{tabular}

Image size $256 \times 256$

\begin{tabular}{|c|c|c|c|c|c|c|c|c|}
\hline house & 1.24 & 31.34 & 0.322 & 33.938 & 74.5292 & 1.5305 & 31.667 & 15.681 \\
\hline zelda & 1.00 & 32.06 & 0.329 & 34.029 & 72.8886 & 1.6887 & 31.198 & 14.212 \\
\hline \multicolumn{9}{|c|}{ Image size $512 \times 512$} \\
\hline Lena & 1.03 & 32.76 & 0.351 & 33.544 & 68.2333 & 1.6002 & 31.793 & 14.998 \\
\hline Airplane & 0.90 & 31.46 & 0.338 & 34.502 & 70.9736 & 1.3374 & 30.349 & 17.945 \\
\hline Fruit & 1.47 & 30.47 & 0.405 & 33.405 & 59.1970 & 1.3571 & 30.244 & 17.685 \\
\hline Average & 1.128 & 31.618 & 0.349 & 33.8836 & 69.16434 & 1.50278 & 31.0502 & 16.1042 \\
\hline
\end{tabular}

Fig. 7 Comparison between the proposed method (A-3), CBDCT-CABS [18] and JPEG method on test images

\section{REFERENCES}

[1] Yan Wang; Shoushun Chen; Bermak, A., "FPGA Implementation of Image Compression using DPCM and FBAR," in International Conference on Integrated Circuits, (ISIC '07), 2007, pp. 329-332.

[2] Xiwen OwenZhao, Zhihai HenryHe, "Lossless Image Compression Using Super-Spatial Structure Prediction," IEEE Signal Processing Letters, vol. 17, no. 4, April 2010.

[3] Nelson M. The Data Compression Book. 2nd ed. New York: M\&T Books 1995.

[4] Michael B. Martin and Amy E. Bell, "New Image Compression Techniques Using Multiwavelets and Multiwavelet Packets," IEEE Transactions on Image Processing, vol. 10, no. 4, Apr 2001.

[5] Aaron T. Deever and Sheila S. Hemami, "Lossless Image Compression With Projection-Based and Adaptive Reversible Integer Wavelet Transforms," IEEE Transactions on Image Processing, vol. 12, no. 5, May 2003.
[6] Nikolaos V. Boulgouris, Dimitrios Tzovaras, and Michael Gerassimos Strintzis, "Lossless Image Compression Based on Optimal Prediction, Adaptive Lifting, and Conditional Arithmetic Coding," IEEE Transactions on Image Processing, vol. 10, no. 1, Jan 2001

[7] Eddie Batista de Lima Filho, Eduardo A. B. da Silva Murilo Bresciani de Carvalho, and Frederico Silva Pinagé, "Universal Image Compression Using Multiscale Recurrent Patterns With Adaptive Probability Model," IEEE Transactions on Image Processing, vol. 17, no. 4, Apr 2008.

[8] Xin Li and Michael T. Orchard "Edge-Directed Prediction for Lossless Compression of Natural Images," IEEE Transactions on Image Processing, vol. 10, no. 6, Jun 2001.

[9] J. Wang, K.Y. Min, J.W. Chong, "Cost Effective Block Truncation Coding for Color Image Compression," Advanced in Information Sciences and Service Sciences, vol. 2, no. 3, September 2010, pp.91-98.

[10] K.Sowmyan, A.Siddarth, D.Menaka, "A Novel Approach to Image Compression of Colour Images by Plane Reduction Technique," World 
World Academy of Science, Engineering and Technology

International Journal of Computer and Information Engineering

Vol:8, No:2, 2014

Academy of Science, Engineering and Technology 81, 2011, pp. 292295.

[11] Satish Kumar Singh, Shishir Kumar, "Novel adaptive color space transform and application to image compression," Signal Processing: Image Communication 26, 2011, pp. 662-672.

[12] Arash Abadpour, Shohreh Kasaei, "Color PCA Eigenimages and their Application to Compression and Watermarking", submitted to Image \& Vision Computing 21 August 2007.

[13] Davis. L, "Survey of edge detection techniques, computer vision", Graph. Image Process, vol. 4, 1975, pp. 248-270.

[14] Canny. J, "A computational approach to edge detection," IEEE Trans. Pattern Anal. Machine. Intel., vol. PAMI-8, Nov. 1986, pp. 679-698.

[15] Pennebaker, W.B. Mitchell, J.L. Langdon, G.G. Arps Jr., R. B. (1988), "An overview of the basic principles of the Q-Coder adaptive binary arithmetic coder," IBM Journal of Research and Development, vol. 32, no. 6,1988 .

[16] Amhamed Saffor, Abdul Rahman Ramli, Kwan-Hoong Ng, "A Comparative Study of Image Compression between JPEG and Wavelet," Malaysian Journal of Computer Science, vol. 14, no. 1, June 2001, pp 39-45.

[17] Walaa M. Abd-Elhafiez, Wajeb Gharibi, "Color Image Compression Algorithm Based on DCT Blocks," International Journal of Computer Science Issues, IJCSI, vol. 9, Issue 4, July 2012, pp. 323-328.

[18] F. Douak, Redha Benzid, Nabil Benoudjit "Color image compression algorithm based on the DCT transform combined to an adaptive block scanning,” Int. J. Electron. Commun. (AEU), vol. 65, 2011, pp. 16-26. 